\title{
Qualidade fisiológica de sementes de milho tratadas com molibdênio ${ }^{1}$
}

\author{
Francisco Rafael da Silva Pereira ${ }^{2 *}$, Elizeu Luiz Brachtvogel ${ }^{2}$, Simério Carlos Silva \\ $\mathrm{Cruz}^{3}$, Silvio José Bicudo ${ }^{4}$, Carla Gomes Machado ${ }^{3}$, Juliana Campana Pereira ${ }^{4}$ \\ RESUMO - O tratamento de sementes com micronutrientes, como o molibdênio, garante \\ uma maior uniformidade de aplicação, sendo que, a quantidade a ser aplicada desse elemento \\ nas sementes deve ser suficiente para provir à exigência para o desenvolvimento e produção \\ da cultura. Assim, objetivou-se com o presente trabalho avaliar a qualidade fisiológica de \\ sementes de milho tratadas com molibdênio. A qualidade das sementes foi avaliada por meio \\ da determinação do teor de água, do teste de germinação, da primeira contagem de germinação \\ e da emissão de raízes primárias. Os tratamentos testados consistiram de cinco híbridos (DOW \\ CO32; DOW 2B587; DOW 2B688; PIONEER 30F35 e PIONEER 30K73) e cinco doses de \\ molibdênio aplicadas via semente $\left(0 ; 7,5 ; 22,5 ; 67,5 ; 202,5 \mathrm{~g} \mathrm{ha}^{-1}\right.$ de molibdênio). A fonte de \\ molibdênio utilizada foi o molibdato de sódio dihidratado (39\% de molibdênio), sendo que a \\ aplicação do molibdênio foi efetuada por meio da mistura com o fungicida líquido de suspensão \\ concentrada carboxina+thiram sobre as sementes. A qualidade fisiológica das sementes de \\ milho é influenciada negativamente por doses crescentes de molibdênio aplicadas. O híbrido de \\ milho DOW 2B587 obteve melhor resposta à aplicação da maior dose de molibdênio em relação \\ aos demais híbridos estudados.
}

Termos para indexação: molibdato de sódio, efeito salino, híbridos.

\section{Physiological quality of corn seeds treated with molybdenum}

\begin{abstract}
Seed treatment with micronutrients, such as molybdenum, ensures a greater uniformity of application. The amount of this element to be applied to seeds must be sufficient for crop development and production. The objective of the present study was to evaluate the physiological quality of corn seeds treated with molybdenum. Seed quality was evaluated by determining the water content, and from the germination test, the first count test and primary root emission. The treatments consisted of five hybrids (DOW CO32; DOW 2B587; DOW 2B688; Pioneer 30F35 and Pioneer 30K73) and five levels of molybdenum applied to the seeds $(0 ; 7.5$; $22.5 ; 67.5 ; 202.5 \mathrm{~g} \mathrm{ha}^{-1}$ of molybdenum). The source of molydenum was sodium molybdate dyhidrate (39\% of molybdenum) and the application was made by mixing with the liquid suspension of a carboxim+thiram fungicide on the seeds. The physiological quality of the corn seeds is adversely affected by increasing levels of molybdenum. The DOW 2B587 corn hybrid gave the best response to the application of the highest level of molybdenum compared to the other genotypes studied.
\end{abstract}

Index terms: sodium molybdate, saline effect, hybrids.

Submetido em 17/02/2011. Aceito para publicação em 05/01/2012.

${ }^{2}$ Instituto Federal de Educação, Ciência e Tecnologia de São Paulo, 01109-010-São Paulo, SP, Brasil.
${ }^{4}$ Departamento de Produção Vegetal, FCA/UNESP, Caixa Postal 237, 18610-307-Botucatu, SP, Brasil.

*Autor para correspondência < pereirafrs@hotmail.com>

${ }^{3}$ Universidade Estadual de Goiás, 76190-000-Palmeiras de Goiás, GO, Brasil. 


\section{Introdução}

O molibdênio é um dos micronutrientes exigidos em menor quantidade pelas plantas. Apesar disso, muitas pesquisas têm apresentado respostas interessantes à aplicação de molibdênio em diferentes culturas (Srivastava, 1997), inclusive no milho, indicando possíveis limitações no suprimento deste elemento pelos solos brasileiros (Fancelli e Dourado Neto, 2000).

No metabolismo das plantas uma de suas principais funções é o indispensável papel que o molibdênio exerce na assimilação do nitrato absorvido pelas plantas, atuando na enzima nitrato redutase. Portanto, qualquer deficiência desse elemento pode comprometer o metabolismo do nitrogênio, diminuindo o rendimento das culturas (Malavolta, 2006). O molibdênio utilizado pelas plantas pode ser originado do próprio solo ou resultante da aplicação de produtos químicos e, ou, orgânicos que o contenham em sua composição. Albino e Campo (2001) relatam que uma das principais fontes de molibdênio é o molibdato de sódio.

$\mathrm{O}$ tratamento de sementes com micronutrientes tem como princípio a translocação desses micronutrientes aplicados na semente para a futura planta (Cheng, 1985). A uniformidade de distribuição de pequenas doses que podem ser aplicadas com precisão é uma das grandes vantagens desse método de aplicação (Mortvedt, 1997). Essa é uma tecnologia de comprovada eficiência na aplicação de molibdênio e também de cobalto em leguminosas, com vista à fixação simbiótica de nitrogênio. Em soja, por exemplo, a recomendação atual de aplicação do molibdênio é via sementes antes da aplicação do Bradyrhizobium (Ferreira et al., 2003).

$O$ tratamento de sementes garante uma maior uniformidade de aplicação no campo e a quantidade de molibdênio que pode ser aplicado nas sementes é suficiente para provir à exigência de crescimento da cultura (Mortvedt, 1997). As doses recomendadas para o tratamento de sementes variam de 7 a $100 \mathrm{~g} \mathrm{ha}^{-1}$ de molibdênio (Marschner, 1995; Srivastava e Gupta, 1996), pois doses elevadas podem ser tóxicas. De acordo com Pessoa et al. (2000), além da toxicidade, sabe-se também, que concentrações altas de sais próximas à semente podem prejudicar a emergência das plântulas.

Apesar da comprovada eficiência desse método de aplicação, assim como ocorre com o fornecimento via solo, a aplicação direta na semente poderá ter sua eficiência comprometida pelo processo de imobilização pela matéria orgânica, óxidos de ferro e de alumínio, o que resultaria na redução da disponibilidade do molibdênio para as plantas (Guerra et al., 2006). Porém, em solos ácidos da África, Tanner e Grant (1977) registraram efeito positivo da aplicação desse micronutriente, em tratamento das sementes, sobre a produção do milho.

Face ao exposto, objetivou-se com o presente trabalho avaliar a qualidade fisiológica de sementes de milho tratadas com molibdênio.

\section{Material e Métodos}

O experimento foi conduzido no Laboratório de Análise de Sementes, do Departamento de Produção Vegetal Agricultura, da Faculdade de Ciências Agronômicas Universidade Estadual Paulista (UNESP), Campus de Botucatu.

Foi empregado o delineamento experimental inteiramente casualizado, com quatro repetições em esquema fatorial $5 \times 5$, sendo cinco híbridos (CO32; 2B587; 2B688; 30F35 e 30K73) e cinco doses de molibdênio aplicadas via semente $(0 ; 7,5$; 22,5; 67,5; 202,5 $\mathrm{g} \mathrm{ha}^{-1}$ de molibdênio).

A fonte de molibdênio utilizada foi o molibdato de sódio dihidratado (39\% de molibdênio). A aplicação do molibdênio via semente foi efetuada por meio da mistura com o fungicida líquido de suspensão concentrada carboxina+thiram sobre as sementes. Foram aplicados $300 \mathrm{~mL}$ do produto comercial / $100 \mathrm{~kg}$ de sementes, seguindo a recomendação do fabricante (Chemtura, 2008). Para a aplicação do fungicida na dose recomendada pelo fabricante e das doses de molibdênio, primeiramente foram realizadas determinações da massa de mil sementes de cada híbrido segundo Brasil (2009), que tiveram suas massas determinadas e ajustadas para $13 \%$ de teor de água, possibilitando estimar a massa de 1000 sementes (Tabela 1). Em seguida, foi estimada a massa de sementes de cada híbrido equivalente a um hectare $(60.000$ sementes). A partir desses valores foram calculadas a dose do fungicida e as doses de molibdato de sódio.

Tabela 1. Massa de 1000 sementes em gramas (g) de cinco híbridos de milho.

\begin{tabular}{cc}
\hline Híbrido & $\begin{array}{c}\text { Massa de } 1000 \text { sementes } \\
(\mathrm{g})\end{array}$ \\
\hline DOW CO32 & 285,4 \\
DOW 2B688 & 326,6 \\
DOW 2B587 & 319,0 \\
Pioneer 30F35 & 264,0 \\
Pioneer 30K73 & 278,4 \\
\hline
\end{tabular}


O tratamento das sementes foi efetuado com 100 sementes por aplicação em Becker de vidro, agitandoas com movimentos rotativos para possibilitar uma distribuição homogênea do produto. Para a caracterização da qualidade das sementes foram realizados os testes descritos a seguir.

Teor de água: Antes da aplicação dos tratamentos, o teor de água foi determinado pelo método da estufa a $105^{\circ} \mathrm{C}$ por 24 horas, utilizando-se duas subamostras de 25 sementes (Brasil, 2009).

Germinação: Foi realizada com oito subamostras de 50 sementes por lote, em rolo de papel toalha (RP), previamente umedecido com água destilada na proporção de 2,5 vezes a massa do substrato, à temperatura de $30{ }^{\circ} \mathrm{C}$, determinando-se a porcentagem de plântulas normais, avaliadas no quarto e sétimo dias após a instalação do teste (Brasil, 2009). Visando a manutenção da umidade do substrato, os rolos de papel toalha foram acondicionados em sacos plásticos conforme metodologia desenvolvida por (Coimbra et al. 2007).

Primeira contagem da germinação: Foram avaliadas as plântulas normais no quarto dia do teste de germinação com resultados expressos em porcentagem (Brasil, 2009).

Precocidade de emissão de raiz primária: Realizado com oito subamostras de 50 sementes, em rolo de papel toalha, previamente umedecido com água destilada na proporção de 2,5 vezes a massa do substrato, à temperatura de $30{ }^{\circ} \mathrm{C}$. As contagens foram realizadas trinta e duas horas após a instalação do teste, contabilizando-se a porcentagem de sementes que emitiram raiz primária (Toledo et al., 1999).

Os dados foram submetidos à análise de variância. As médias obtidas para os híbridos, foram comparadas pelo teste de médias (DMS) a 5\% de significância. Para a variável dose, foram realizadas análise de regressão, calculada para equações lineares e quadráticas. Os parâmetros que apresentaram dados em porcentagem com valores nulos foram transformados em $\sqrt{ }(x+0,5)$, para realização das análises estatísticas (Barbin, 2003). As médias apresentadas nas tabelas e figuras referem-se aos dados originais.

O cálculo da máxima dose de molibdênio, aquela em que a germinação não foi reduzida abaixo de $85 \%$, foi realizada igualando-se a equação da germinação de cada híbrido a 85 e resolvendo esta através da fórmula Bháskara.

\section{Resultados e Discussão}

Na Tabela 2 são apresentados os valores de F calculados para as fontes variação e suas interações dos parâmetros teor de água das sementes, precocidade de emissão de raiz primária, primeira contagem e germinação.

Tabela 2. Resumo da análise de variância (valores de F) para as causas de variação: híbrido (H), dose (D) e suas interações (H*D) da variável teor de água das sementes (TA), precocidade de emissão de raízes primárias (PERP), primeira contagem (PC) e germinação $(\mathrm{G})$.

\begin{tabular}{ccccc}
\hline Análise de & \multicolumn{3}{c}{ Variável analisada } \\
\cline { 2 - 5 } variância & TA & PERP & PC & G \\
\hline H & $2,503^{\text {ns }}$ & $76,212^{* *}$ & $38,438^{* *}$ & $14,809^{* *}$ \\
$\mathrm{D}$ & - & $224,899^{* *}$ & $849,862^{* *}$ & $389,894^{* *}$ \\
$\mathrm{H}^{*} \mathrm{D}$ & - & $6,832^{* *}$ & $9,673^{* *}$ & $6,195^{* *}$ \\
\hline CV $\%$ & 5,1 & 18,29 & 12,82 & 17,52 \\
\hline & & & Regressão Polinomial & \\
\hline Reg. linear & - & $756,515^{* *}$ & $2.139,499^{* *}$ & $1.043,617^{* *}$ \\
Reg. quadrática & - & $138,490^{* *}$ & $1.206,047^{* *}$ & $501,701^{* *}$ \\
\hline
\end{tabular}

** Significativo a $1 \%$ de probabilidade $(\mathrm{p}<0,01)$; Significativo a $5 \%$ de probabilidade $(0,01=<\mathrm{p}<0,05)$; ${ }^{\text {ns }}$ Não significativo (p $\left.>=0,05\right)$. CV $\%$ coeficiente de variação

Nota-se que não houve diferença no teor de água das sementes entre os cinco híbridos utilizados no experimento. Considerando-se que o teor de água inicial é um fator primordial para a padronização das avaliações a serem realizadas posteriormente, esses resultados asseguram a credibilidade dos dados obtidos no trabalho. Recomenda-se que amostras de sementes a serem comparadas não apresentem diferenças maiores que $2 \%$ de teor de água, para não comprometerem os resultados devido às diferenças na velocidade de umedecimento das sementes durante o teste (Marcos-Filho, 1999).

Observa-se também na Tabela 2 quanto aos parâmetros de qualidade fisiológica que há diferenças de vigor e poder 
germinativo entre os híbridos testados e que há influência das doses de molibdênio sobre a qualidade fisiológica das sementes.

A Tabela 3 apresenta os resultados do Teste de $\mathrm{T}$ da precocidade de emissão de raiz primária (PERP), primeira contagem (PC) e germinação (G). Para o teste de vigor de precocidade de emissão de raiz primária (PERP), o híbrido CO32 obteve maior PERP em relação aos demais e o material 30 K73 apresentou a menor média. No teste de vigor de primeira contagem da germinação, os híbridos com maiores valores foram: $\mathrm{CO} 32,2 \mathrm{~B} 587$ e 2B688, os quais tiveram comportamento semelhante e assim como no teste de PERP o material 30K73 apresentou a menor média. Quanto à porcentagem de germinação, verifica-se que todos os híbridos estiveram bem abaixo do valor mínimo estipulado para a comercialização de sementes de milho, que é de $85 \%$ (Brasil, 2005). Constata-se também, que o híbrido 2B587 apresentou germinação superior aos demais híbridos testados.

Tabela 3. Valores médios de precocidade de emissão de raiz primária (PERP), primeira contagem $(\mathrm{PC}) \mathrm{e}$ germinação $(\mathrm{G})$ obtidos para os híbridos.

\begin{tabular}{crrr}
\hline \multirow{2}{*}{ Híbrido } & PERP & PC & G \\
\cline { 2 - 4 } & \multicolumn{3}{c}{$\%$} \\
\hline CO32 & $59,4 \mathrm{a}$ & $44,0 \mathrm{a}$ & $46,4 \mathrm{~b}$ \\
$2 \mathrm{~B} 587$ & $33,0 \mathrm{c}$ & $45,4 \mathrm{a}$ & $59,6 \mathrm{a}$ \\
$2 \mathrm{~B} 688$ & $50,3 \mathrm{~b}$ & $47,4 \mathrm{a}$ & $52,1 \mathrm{~b}$ \\
30F35 & $37,7 \mathrm{c}$ & $34,9 \mathrm{~b}$ & $45,8 \mathrm{~b}$ \\
$30 \mathrm{~K} 73$ & $7,3 \mathrm{~d}$ & $21,0 \mathrm{c}$ & $32,3 \mathrm{c}$ \\
\hline
\end{tabular}

Médias seguidas de letras diferentes na coluna diferem entre si em 1\% de probabilidade pelo teste $\mathrm{T}$ (DMS).

Segundo Torres (1998), os testes de vigor são importantes, pois têm alta correlação com a emergência de plântulas em campo, sendo possível, assim, prever o desempenho destas sementes antes da semeadura. A deterioração das sementes é definida, em geral, como toda transformação degenerativa que ocorre nas sementes (Abdul-Baki e Anderson, 1972). Essas transformações deletérias podem ser de origem bioquímica, física ou fisiológica (Woodstock e Grabe, 1967; Ching e Schoolcraft, 1968).

O teste de vigor por meio da primeira contagem baseia-se no fato da velocidade da germinação ser um dos primeiros parâmetros a ser afetado no processo de deterioração das sementes (Nakagawa, 1999). Utilizandose o mesmo princípio, o vigor de um lote de sementes de milho é realizado por meio da avaliação da precocidade de emissão da raiz primária (Toledo et al., 1999). Esses testes apresentam as vantagens de serem econômicos, práticos e simples. Segundo Toledo et al. (1999), a precocidade de emissão da raiz primária mostrou-se viável para estimar o vigor de sementes de milho, uma vez que ela associa a quantidade de sementes que emitem a raiz primária com a velocidade com que este processo ocorre.

Para Delouche e Baskin (1973), os eventos que ocorrem durante a deterioração se iniciam com a desorganização e perda do controle da permeabilidade das membranas das sementes, fatos estes que culminam com a queda de germinação e a morte do embrião. Esse fato pôde ser observado na presente pesquisa, onde o híbrido 30K73 obteve menor vigor e, consequentemente, menor porcentagem de germinação (Tabela 3 ).

Nas Figuras 1, 2 e 3 são apresentados, respectivamente, os resultados da precocidade de emissão de raízes primárias, primeira contagem da germinação e germinação de cinco híbridos de milho em função das doses crescentes de molibdênio. Nota-se que estes parâmetros foram influenciados negativamente pelas doses crescentes de molibdênio, provavelmente ocasionados por danos causados no embrião da semente devido às altas concentrações de sal na semente.

Tem sido relatado em diversos trabalhos com aplicação de micronutrientes via tratamento de sementes, e observado em condições de campo por agricultores, que altas concentrações de sal, como o molibdato, próximo à semente, diminui e atrasa a germinação, reduzindo o estande de lavouras e consequentemente a produtividade (Pessoa et al., 2000; Gonçalves Júnior e Pessoa, 2002; Luchese et al. 2004). Porém, Ribeiro et al. (1994), testando fontes de zinco e boro na forma de sais e boro da marca comercial Biocrop ${ }^{\circledR}$ aplicadas às sementes de milho, na dose recomendada pelo fabricante, não encontraram diferenças entre a testemunha e as sementes tratadas quanto à germinação.

Verificou-se na presente pesquisa que, mesmo sem aplicação de molibdênio, o híbrido $30 \mathrm{~K} 73$ apresentou valores muito baixos nos testes de vigor (precocidade de emissão de raiz primária e primeira contagem de germinação) e no de porcentagem de germinação, demonstrando problemas de qualidade fisiológica das sementes no lote avaliado desse híbrido. Os demais híbridos apresentaram resultados satisfatórios de vigor e germinação no tratamento sem aplicação de molibdênio, com valores de germinação próximos a $100 \%$ (Figuras 1, 2 e 3 ). 


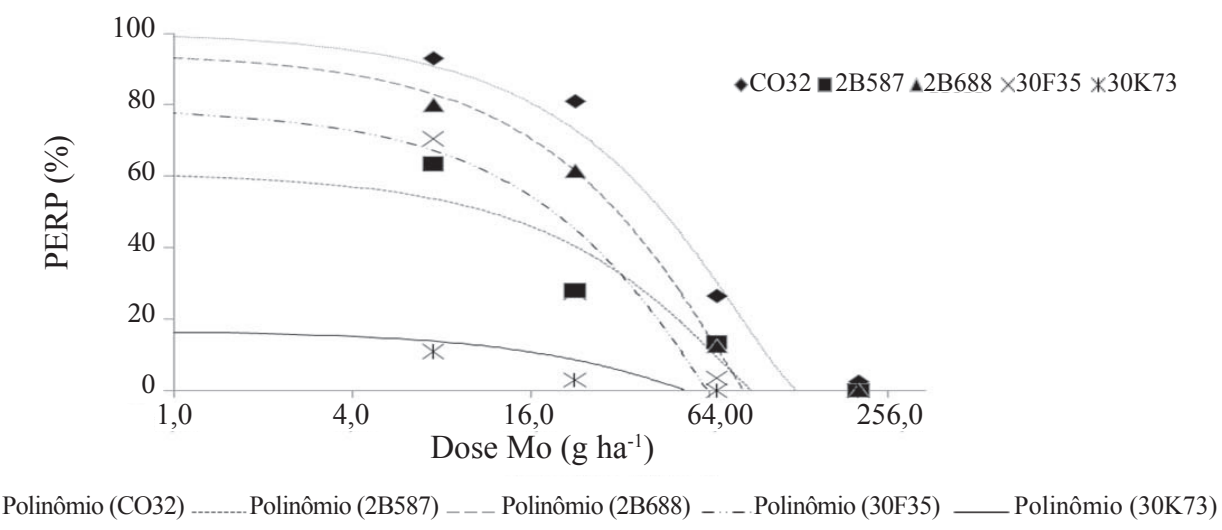

Figura 1. Precocidade de emissão de raízes primárias (PERP) de sementes de cinco híbridos de milho em função de doses crescentes de molibdênio (Mo). CO32 $\left(\mathrm{y}=0,0041 \mathrm{x}^{2}-1,3159 \mathrm{x}+100,54 ; \mathrm{R}^{2}=0,98^{* *}\right), 2 \mathrm{~B} 587\left(\mathrm{y}=0,0034 \mathrm{x}^{2}-0,9934 \mathrm{x}\right.$ $+61,006 ; 0,92 * *), 2 B 688\left(y=0,0056 x^{2}-1,6069 x+94,833 ; R^{2}=1,00 * *\right), 30 F 35\left(y=0,0062 x^{2}-1,6534 x+79,282 ; R^{2}=\right.$ $0,93 * *)$ e $30 \mathrm{~K} 73\left(\mathrm{y}=0,0016 \mathrm{x}^{2}-0,3968 \mathrm{x}+16,74 ; \mathrm{R}^{2}=0,78^{* *}\right)$.

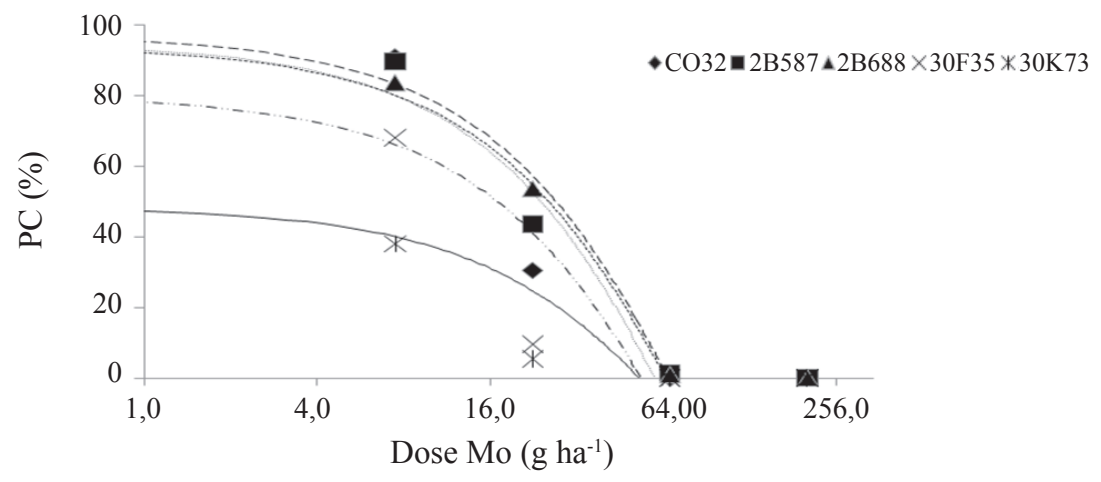

Figura2.Primeiracontagemdagerminação(PC)desementesdecincohíbridosdemilhoemfunçãodedosescrescentesdemolibdênio (Mo). CO32 ( $\left.\mathrm{y}=0,0078 \mathrm{x}^{2}-2,0547 \mathrm{x}+94,881 ; \mathrm{R}^{2}=0,93 * *\right), 2 \mathrm{~B} 587\left(\mathrm{y}=0,0071 \mathrm{x}^{2}-1,8998 \mathrm{x}+93,91 ; \mathrm{R}^{2}=0,97 * *\right), 2 \mathrm{~B} 688(\mathrm{y}=0,0072 \mathrm{x} 2$ $\left.-1,9327 x+97,141 ; R^{2}=1,00 * *\right), 30 F 35\left(y=0,0074 x^{2}-1,886 x+79,85 ; R^{2}=0,81 * *\right)$ e $30 K 73\left(y=0,0045 x^{2}-1,1549 x+48,403\right.$; $\left.\mathrm{R}^{2}=0,80^{* *}\right)$.

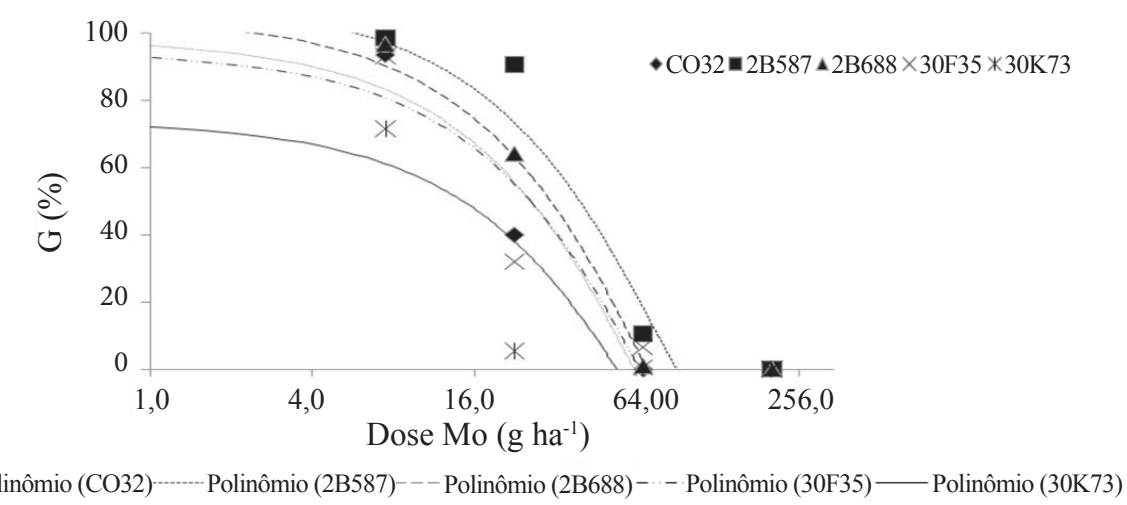

Figura 3. Germinação $(\mathrm{G})$ de sementes de cinco híbridos de milho em função de doses crescentes de molibdênio (Mo). $\operatorname{CO} 32\left(\mathrm{y}=0,0078 \mathrm{x}^{2}-2,0568 \mathrm{x}+98,081 ; \mathrm{R}^{2}=0,96^{* *}\right), 2 \mathrm{~B} 587\left(\mathrm{y}=0,006 \mathrm{x}^{2}-1,7615 \mathrm{x}+109,92 ; \mathrm{R}^{2}=0,95^{* *}\right), 2 \mathrm{~B} 688(\mathrm{y}=$ $\left.0,0074 x^{2}-2,019 x+104,89 ; R^{2}=0,99 * *\right), 30 F 35\left(y=0,0071 x^{2}-1,9069 x+94,445 ; R^{2}=0,91 * *\right)$ e $30 K 73\left(y=0,0068 x^{2}-\right.$ $\left.1,7307 \mathrm{x}+73,63 ; \mathrm{R}^{2}=0,79 * *\right)$. 
Na Tabela 4, são apresentadas as doses máximas de molibdênio, em $\mathrm{g} \mathrm{ha}^{-1}$ aplicado via tratamento de sementes, que os híbridos de milho suportam sem reduzir a germinação abaixo de $85 \%$, cujo valor é o mínimo estipulado para a comercialização de sementes de milho (Brasil, 2005). Verificou-se nos resultados obtidos que o híbrido 2B587 tolera dose de molibdênio mais elevada (14,9 $\mathrm{g} \mathrm{ha}^{-1}$, valor equivalente a $0,73 \mathrm{~g} \mathrm{~kg}^{-1}$ de sementes) em relação aos demais híbridos testados.

Tabela 4. Dose máxima de molibdênio na forma de molibdato de sódio ( $85 \%$ de germinação), aplicado via tratamento de sementes, suportada por sementes de híbridos de milho.

\begin{tabular}{cc}
\hline \multirow{2}{*}{ Híbrido } & Dose máxima $(85 \%$ de germinação $)$ \\
\cline { 2 - 2 } & $\mathrm{g} \mathrm{ha}^{-1}$ de molibdênio \\
\hline CO32 & 6,6 \\
2B587 & 14,9 \\
2B688 & 10,3 \\
30F35 & 5,1 \\
30K73 & - \\
\hline
\end{tabular}

Pessoa et al. (2000), avaliando a germinação e o desenvolvimento inicial de plantas de milho, em resposta ao tratamento de sementes com boro, encontrou que a dose máxima de boro suportada pelas sementes de milho, sem apresentar atrasos no processo germinativo, foi de $0,04 \mathrm{~g} \mathrm{~kg}^{-1}$. Gonçalves Júnior e Pessoa (2002) testando a germinação de sementes e desenvolvimento inicial de plantas de soja em resposta à aplicação de ferro via semente, verificou que a melhor dose esteve entre 0,8 e $1,1 \mathrm{~g} \mathrm{~kg}^{-1}$ de semente. Luchese et al. (2004), em experimento que avaliou a emergência e absorção de cobre por plantas de milho, em resposta ao tratamento de sementes com cobre, verificou que a dose de 5,09 g de cobre por $\mathrm{kg}$ de sementes limitou, a capacidade de germinação das sementes.

\section{Conclusões}

Aqualidade fisiológica das sementes de milhoé influenciada negativamente por doses crescentes de molibdênio.

O híbrido de milho DOW 2B587 obteve melhor resposta à aplicação da maior dose de molibdênio em relação aos demais híbridos estudados.

\section{Referências}

ABDUL-BAKI, A.A.; ANDERSON, J.D. Physiological and biochemical deterioration of seeds. In: KOZLOWSKI, T.T. (Ed.). Seed biology. New York: Academic Press, 1972. p.283-315.

ALBINO, U.B.; CAMPO, R.J. Efeito de fontes e doses de molibdênio na sobrevivência do Bradyrhizobium e na fixação biológica de nitrogênio em soja. Pesquisa Agropecuária Brasileira, v.36, n.3, p.527-534, 2001. http://www.scielo.br/pdf/pab/v36n3/4797.pdf

BARBIN, D. Planejamento e análise de experimentos agronômicos. Arapongas: Midas, 2003. 208p.

BRASIL. Ministério da Agricultura, Pecuária e Abastecimento. Regras para análise de sementes. Ministério da Agricultura, Pecuária e Abastecimento. Secretaria de Defesa Agropecuária. Brasília, DF: MAPA/ ACS, 2009. 395p. http://www.bs.cca.ufsc.br/publicacoes/regras\%20 analise $\% 20$ sementes.pdf

BRASIL. Ministério da Agricultura, Pecuária e Abastecimento. Padrões para produção e comercialização de sementes de milho - cultivares híbridas. Instrução normativa $\mathrm{n}^{\circ} 25$, de 16 de dezembro de 2005. Diário Oficial da União, Brasília, DF, 20 dez. 2005. Seção 1, n.243, p.18.

CHEMTURA. Vitavax - Thiran 200 SC. http://www.chemtura.com/ deployedfiles/Business\%20Units/Crop_Protection-pt-BR/BU\%20 Documents/Material\%20Safety\%20Data\%20Sheets/VITAVAX\%20 THIRAM\%20200\%20SC.pdf. Acesso em: 02 fev. 2008.

CHENG, T. The effect of seed treatment with microelements upon the germination and early growth of wheat. Science Sinica, v.44, p.129-135, 1985.

CHING, T.M.; SCHOOLCRAFT, I. Physiological and chemical differences in aged seeds. Crop Science, v.8, p.407-409, 1968.

COIMBRA, R. A.; TOMAZ, C. A.; MARTINS, C.C.; NAKAGAWA, J. Teste de germinação com acondicionamento dos rolos de papel em sacos plásticos. Revista Braileira de Sementes, v.29, n.1, p.92-97, 2007. http:// www.scielo.br/pdf/rbs/v29n1/13.pdf

DELOUCHE, J.C.; BASKIN, C.C. Acelerated aging techniques for predicting the relative storability of seed lots. Seed Science and Technology, v.1, n.2, p.427-452, 1973.

FANCELLI, A.L.; DOURADO-NETO, D. A produção de milho. Guaíba: Agropecuária, 2000. 360p.

FERREIRA, A.C.B.; ARAÚJO, G. A. A.; CARDOSO, A.A.; FONTES, P.C.R.; VIEIRA, C. Características agronômicas do feijoeiro em função do molibdênio contido na semente e da sua aplicação foliar. Acta Scientiarium: Agronomy, v.25, n.1, p.65-72, 2003.

GONÇALVES JUNIOR, A.C.; PESSOA, A.C.S. Avaliação da fitodisponibilidade de cádmio, chumbo e crômio, em soja cultivada em Argissolo Vermelho Eutrofico, tratado com fertilizantes comerciais. Scientia Agraria, v.3, n.1/2, p.19-23, 2002.

GUERRA, C.A.; MARCHETTI, M.E.; ROBAINA, A.D.; SOUZA, L.C.F.; GONÇALVES, M. C.; NOVELINO, J.O. Qualidade fisiológica de sementes de soja em função da adubação com fósforo, molibdênio e 
cobalto. Acta Scientarum: Agronomy, v.28, n.1, 2006. p. 91-97. http:// periodicos.uem.br/ojs/index.php/ActaSciAgron/article/view/1678/1043

LUCHESE, A.V.; GONÇALVES JUNIOR, A.C.; LUCHESE, E.B. BRACCINI, M. C. L. Emergência e absorção de cobre por plantas de milho (Zea mays) em resposta ao tratamento de sementes com cobre. Ciencia Rural, v.24, n.6, p.1949-1952, 2004. http://www.scielo.br/pdf/cr/v34n6/a44v34n6.pdf

MALAVOLTA, E. Manual de nutrição mineral de plantas. São Paulo: Editora Agronômica Ceres, 2006. 638p.

MARCOS-FILHO, J. Teste de envelhecimento acelerado. In: KRZYZANOWSKY, F.C.; VIEIRA. R. D.; FRANÇA- NETO, J.B. Vigor de sementes: conceitos e testes. Londrina: ABRATES, 1999. p.3.1-3.24.

MARSCHNER, H. Mineral nutrition of higer plants. 2.ed. New York: Academic Press, 1995. 889p.

MORTVEDT, J.J. Sources and methods for molybdenum fertilization in crops. In: GUPTA, U.C. (Ed.). Molybdenum in agriculture. New York: Cambridge University Press, 1997. p.171-181.

NAKAGAWA, J. Testes de vigor baseados no desempenho das plântulas - primeira contagem. In: KRZYZANOWSKI, F.C.; VIEIRA, R.D.; FRANÇA-NETO, J.B. (Ed.). Vigor de sementes: conceitos e testes. Londrina: ABRATES, 1999. p.2.7-2.8

PESSOA, A.C.S.; LUCHESE, E.B.; LUCHESE, A.V. Germinação e desenvolvimento inicial de plantas de milho, em resposta ao tratamento de sementes com boro. Revista Brasileira de Ciência do Solo, v.24, n.4, p.939-945, 2000. http://sbes.solos.ufv.br/solos/revistas/v24n4a25.pdf
RIBEIRO, N.D.; SANTOS, O.S.; MENEZES, N.L. Tratamento de sementes de milho com fontes de zinco e boro. Revista Brasileira de Sementes, v.16, n.2, p.116-120, 1994. http://www.abrates.org.br/revista/ artigos/1994/v16n2/artigo03.pdf

SRIVASTAVA, P.C. Biochemical significance of molybdenum in crop plants. In.: GUPTA U. C. (Ed.). Molybdenum in agriculture. New York: Cambridge University Press, 1997. p.47-70.

SRIVASTAVA, P.C.; GUPTA, U.C. Trace elements in crop production. Labanon, NH: Science Publishers, 1996. 356p.

TANNER, P.D.; GRANT, P.M. Response of maize (Zea mays L.) to lime and molybdenum on acid red and yellow-brown clay loams. Rhodesian Journal of Agricultural Research, v.15, p.143-149, 1977.

TOLEDO, F.F.; NOVEMBRE, A.D.L.C.; CHAMMA, H.M.C.P.; MASCHIETO, R.W. Vigor de sementes de milho (Zea mays L.) avaliado pela precocidade de emissão da raiz primária. Scientia Agricola, v.56, n.1, p.191-196, 1999. http://www.scielo.br/scielo.php?pid=S010390161999000100026\&script=sci_abstract\&tlng=pt

TORRES, S.B. Testes de vigor na avaliação da qualidade fisiológica de sementes de milho. Revista Brasileira de Sementes, v.20, n.1, p.55-59, 1998. http://www.abrates.org.br/revista/artigos/1998/v20n1/artigo10.pdf

WOODSTOCK, L.W.; GRABE, D.F. Relationship between seed respiration during imbibition and subsequent seedling growth in Zea mays. Plant Physiology, v.8,p.339-342, 1967.http://www.jstor.org/discover/10.2307/42611 07 ?uid $=3737664$ \&uid $=2129$ \&uid $=2$ \&uid $=70$ \&uid $=4 \&$ sid $=47698759904467$ 